\title{
Acupuncture Treatment for Sports Injury - Hamstring Muscles Group
}

\author{
Jihe Zhu ${ }^{1}$, Blagica Arsovska ${ }^{1,2}$, Kristina Kozovska ${ }^{1, *}$ \\ ${ }^{1}$ Faculty of Medical Sciences, University “Goce Delcev", Shtip, Republic of Macedonia \\ ${ }^{2}$ Institute of Biology, Faculty of Natural Sciences and Mathematics, University “St. Cyril and Methodius” Skopje, Republic of Macedonia
}

Email address:

tongdatang-tcm@hotmail.com (K. Kozovska)

${ }^{*}$ Corresponding author

To cite this article:

Jihe Zhu, Blagica Arsovska, Kristina Kozovska. Acupuncture Treatment for Sports Injury - Hamstring Muscles Group. International Journal of Clinical and Experimental Medical Sciences. Vol. 3, No. 6, 2017, pp. 71-73. doi: 10.11648/j.ijcems.20170306.11

Received: September 26, 2017; Accepted: October 26, 2017; Published: November 20, 2017

\begin{abstract}
Biceps femoris is one of the muscles that forms the hamstring muscles group. Most of the athletes of all ages and sports and some active individuals, happen to have hamstring strain as most common injury in the group of active individuals. From the Chinese medical point of view, human body is not only formed of mechanic parts, but it's an energetic collection of functions. We have the Life Energy within us, which is known as Qi. If a person has an injury, around the injured area the flow of energy becomes disrupted, causing pain and stagnation. The hamstring strain is at the level of muscles and tendons, with stagnation of the Qi and blood in channels and collaterals. The main purpose of doing this work is to show the positive results and effects of acupuncture. In the research are included 8 professional athletes in different sports, male and female, on age from 17 to 55, all with symptoms of biceps femoris pain. All patients were treated with acupuncture, indoor, on room temperature, with duration of treatments of 25-30 minutes. All of them needed only one treatment, expect for one female handball player, who needed 16 treatments to achieve positive result.
\end{abstract}

Keywords: Hamstring, Traditional Chinese Medicine, Acupuncture, Treatment, Athletes

\section{Introduction}

Hamstring injuries are very common in athletes of all ages and sport areas that require running, like football and handball, and also require fast acceleration and deceleration. Hamstrings group of muscles include three muscles - the semimembranosus, the semitendinosus and the biceps femoris muscle. Because they are large group of muscles at the back of the thigh, one injury can affect a large area. A hamstring injury can be rated from 1 to 3 . In a grade 1 there is a minor swelling, stiffness, pain and minor tearing and injured patient can still walk. In grade 2 tearing of the muscle is partial and the patient would have problem to straighten their knee. In a grade 3, there is major or complete tearing of the muscle. [1]

Symptoms of hamstring injuries include tightness, tenderness under the buttock, swelling, spasm and if there is a bigger injury the bruises appears. [2] The Western Medicine suggestsRICE (Rest, Ice, Compressionand,
Elevation) method treatment after injury. Further in the course of rehabilitation, physiotherapy and a range of other physical treatments are applied to help maintain a proper and complete functioning. The studies done in the last 10 years show that the ice is not an appropriate treatment for this pain. [3] The West likes to use ice which is opposite of the East, never uses ice, but opposite, only heat. Also the East accepts inflammatory process as a part of healing process and the West wants to fight inflammation. [4]

Acupuncture for hamstring injury can greatly accelerate the recovery. By the philosophy of Traditional Chinese Medicine (TCM), there is a system of energy meridians which can be utilized to treat a hamstring injury. This energy runs up the back of the leg and to be treated is used a traditional technique known as gua sha. In addition, local acupuncture points specifically on the fibrous tissue are palpated and needled directing the energy away from the injured tissue. [5] 


\section{Material and Methods}

In this article is shown a group of 8 patients, all athletes with same injury of hamstring group of muscles, 2 femafe and 6 male, on age from 17 to 55. All of them needed only one treatment, except for one young female handball player, who needed 16 treatments to solve the problem. This patient also had pain in the lumbal part which is reflected in biceps femoris muscle. All the athletes were treated with acupuncture on the same acupoints, indoor on the roomtemperature with duration of the treatnent of 25 to 30 minutes. Acupuncture treatments were done in a clinic for TCM and acupuncture in Skopje, by a doctor specialist in acupuncture. During the treatments are used fine, sterile acupuncture needles size 0.30 x $40 \mathrm{~mm}$ produced by Wuijuiang City Medical \& Health Material Co., LTD. Acupuncture points that were treated are: Bl 26 (Guan Yua Shu), B1 36 (Chengfu), B1 37 (Yinmen), Bl 38 (Fuxi), B1 56 (Cheng Ying).

\section{Results}

All of the athletes have same injury on biceps femoris muscle which is part of hamstring group of muscles. The pain at the back of the thigh and tightness were present in all of them. Othere symptoms were: tenderness under the buttock, swelling, and spasam. Most of them had pain in the right leg ( 5 patients right and 3 left leg). Of the tretated athlets 6 were male and 2 female. All the patients were professionals at different kinds of sports, three of them were handball players ( 1 female and 2 male), three football players all of them male, one marathoner - male and one karate player - female. The same analysis are shown on Table 1.

Table 1. Differentiation by sport and gender.

\begin{tabular}{lll}
\hline Sport & Male & Female \\
\hline Handball & 2 & 1 \\
Football & 3 & 0 \\
Marathon & 1 & $/$ \\
Karate & $/$ & 1 \\
\hline
\end{tabular}

According to the different age groups and the number of athletes in each group, the most common age group was from 30 to 40 years. There were two athletes in the group from 10 to 20 years, also two athletes in the group from 20 to 30 years, three athletes in the 30 to 40 years age group, no athletes in the group from 40 to 50 years and only one athlete in the group of more than 50 years. The same analysis are shown in Table 2.

Table 2. Age groups

\begin{tabular}{ll}
\hline Age & Athlets \\
\hline $10-20$ & 2 \\
$20-30$ & 2 \\
$30-40$ & 3 \\
$40-50$ & $/$ \\
$>50$ & 1 \\
\hline
\end{tabular}

The causes for hamstring injuries were different like: no warm-up before training, poor stretching, doing some activities which involve rapid acceleration or deceleration such as jumping, sprinting etc. However, after the treatment all the athletes felt better, without pain and other symptoms and were satisfied for returning healthy function to injured parts.

\section{Discussion}

Acupuncture is natural, non-surgical and drug free health care system which provide immediate relief and long lasting benefits. Acupuncture improves circulation, function and mobility, reduces inflammation, relieves stress, helps to alleviate the pain, stimulates local healing response, prevents future injuries, enhances the athletic endurance and performance and by increasing local circulation it helps to improve the joint mobility, muscle stiffness and disperse the swelling. [6] [7] [8] These days, the number of professional athletes and sport individuals who seek acupuncture treatment is constantly increasing. They visit acupuncturists to help optimize their conditioning and for sport injuries. Acupuncture can help with acute and chronic sport injuries. Strains and sprains of the joint and surrounding tissue are most common injuries in the athletes. Beside the pain there might be present skin discoloration, redness, swelling or reduced range of motion. When the RICE method doesn't work, the acupuncture treatment can be used anytime to help to alleviate and ultimately get rid of the pain. [9] In fact, acupuncture not only that is effective in the treatment of acute injuries as sprains, strains, shoulder, elbow, wrist pain and swollen muscles, but it is also very effective in building up any weakness in the body, increasing the recovery time and improving the performances. [7] Human body has over 2000 specific areas known as acupuncture points which are connected through the meridians. TCM practitioners discovered these points on the body thousands of years ago. [10] All the points which were chosen in the treatment were located on the meridians where the energy was blocked, thus to help the blood flow freely and remove all the blockages which cause pain. The hamstrings are connected to the Bladder meridian and when there is some hamstring dysfunction, it affects the Bladder meridian and points that are located on this meridian should be treated. [11]

B1 26 (Guan YuaShu) - Located 1.5 cun lateral to GV line, level L5. It is used to strengthen the lower back, move the Qi and blood in the lower burner and benefit urination. Indications are low back pain, pain in the lower back and buttock, abdominal masses, enuresis and etc.

Bl 36 (Chengfu) - it is located at the mid-point of the base of the buttock. It is used to remove channel obstruction (Bi syndrome) and to treat hemorrhoids. Indications are sciatica, lumbar or spinal pain and hemorrhoids.

B1 37 (Yinmen) - Located on the posterior midline of the leg, 6 cun below the base of the buttocks. It benefits lumbar and sacral regions. Indications are pain in the back, sciatica or sacral pain.

B1 38 (Fuxi) - Located 1 cun above the popliteal line, at 
the medial border of the biceps femoris. It benefits dorsal thigh. Indication is pain in the posterior thigh.

B1 56 (Cheng Ying) - This point is located 6 cun below popliteal line center. It relaxes muscles and tendons of the leg and treats hemorrhoids. Indications are pain in the leg, ankle and foot, cramp in the legs and hemorrhoids.

\section{Conclusion}

Acupuncture as a treatment for sport injuries, specifically hamstrings injuries, is an effective method for acute and chronic pain and gives excellent results for a very short time.

\section{References}

[1] Kinetic health; Resolving Hamstring Injuries; 2012 [www.kinetichealth.ca].

[2] Shiel C. W.; Hamstring Injuries; 2016 [www.medicinenet.com].

[3] Zippelius K., Schwarzinger A.; Sports injuries - better results with integrative traditional Chinese medicine (TCM) therapy; 2015 [www.safflower.com.au].

[4] Bolz M. S.; Sports Injury - Quick Recovery Of Sports Injuries With Traditional Chinese Medicine; 2016 [www.acupunctureplus.us].

[5] Lade H.; Acupuncture for Hamstring Injury; 2012 [www.theacupunctureclinic.co.nz].

[6] Chinese Acupuncture \& Herb Center; Acupuncture and Traditional Chinese Medicine to Heal Chronic and Acute Pain; 2016 [www.chineseacupunctureherbcenter.com].

[7] Fuchs J; Acupuncture for Sports Injury; 2017 [www.vibenaturalhealth.com.au].

[8] Balusik D; Acupuncture for sports injuries; 2008 [www.acupuncturebydawn.com].

[9] Boyle K; Acupuncture For Sports Injuries; 2016 [www.acupunctureinvermont.com].

[10] Waite L.; The Benefits of Acupuncture Therapy for Athletes; 2016 [www.active.com].

[11] Boyd D; Acupuncture Treatment of the Hamstrings; Acupuncture Today; Vol. 03, Issue 02; 2002. 